\title{
Perfil do Consumo de Álcool e drogas ilícitas entre adolescentes ESCOLARES DE UMA CAPITAL BRASILEIRA
}

\author{
Edson Arantes Faria Filho ${ }^{1}$
}

Este artigo descreve e analisa a exposição de adolescentes escolares às bebidas alcoólicas e drogas ilícitas a partir dos dados da Pesquisa Nacional de Saúde do Escolar (PeNSE) 2012. Traçamos o perfil do consumo dessas substâncias na cidade de Goiânia-GO, conforme o sexo e tipo de escola. O percentual de experimentação precoce, consumo atual de bebidas alcoólicas e episódios de embriaguez foram ligeiramente maiores entre as meninas. Nas escolas privadas, o percentual de experimentação de bebidas alcoólicas foi maior e nas escolas públicas o de embriaguez foi maior. Os estudantes do sexo masculino e de escolas públicas apresentaram maior percentual de experimentação de drogas ilícitas. Esses resultados geram evidências para orientar as políticas públicas de saúde voltadas aos adolescentes.

Descritores: Adolescente; Alcoolismo; Drogas Ilícitas; Saúde Escolar.

${ }^{1}$ MSc, Especialista em Saúde, Secretaria Municipal de Saúde, Goiânia, GO, Brasil.

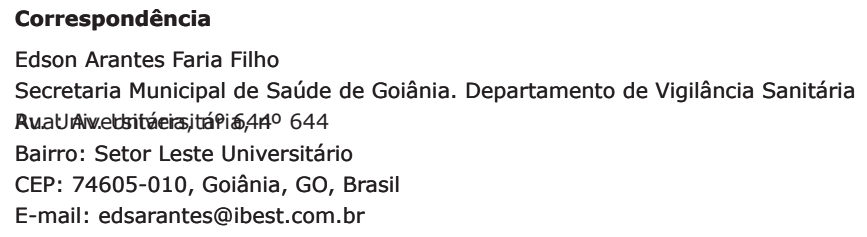




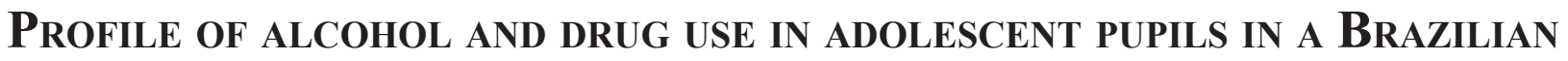 STATE CAPITAL}

This article describes and analyzes exposure of adolescent pupils to alcoholic beverages and illegal drugs based on data from the National Schoolchildren's Health Survey (PeNSE) 2012. We outline the profile of consumption of these substances in the city of Goiânia-GO, according to sex and type of school. The percentage of experimentation at an early age, current alcohol consumption and episodes of drunkenness were slightly higher among girls. In the private schools, the percentage of those who had tried alcohol was higher whereas in the public schools the percentage of drunken episodes was higher. There was a higher percentage of experimentation with illegal drugs among male students and those from public schools. These results produce evidence that can guide public policies aimed at adolescents.

Descriptors: Adolescent; Alcoholism; Street Drugs; School Health.

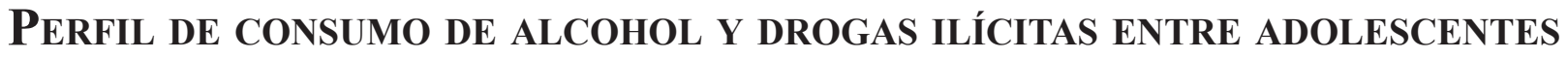 ESCOLARES DE UNA CAPITAL BRASILEÑA}

Este artículo describe y analiza la exposición de adolescentes escolares a las bebidas alcohólicas y drogas ilegales a partir de los datos de la Investigación Nacional de Salud del Escolar (PeNSE) 2012. Delineamos el perfil de consumo de esas substancias en la ciudad de Goiânia-GO, según el sexo y tipo de escuela. El porcentaje de consumo precoz, consumo actual de bebidas alcohólicas y episodios de embriaguez fueron ligeramente mayores entre las chicas. En los colegios privados, el porcentaje de consumo inicial de bebidas alcohólicas fue mayor y en los colegios públicos el de embriaguez fue mayor. Los estudiantes de sexo masculino y de colegios públicos presentaran mayor porcentaje de consumo inicial de drogas ilegales. Esos resultados generan evidencias para orientar las políticas públicas de salud centradas en los adolescentes.

Descriptores: Adolescente; Alcoholismo; Drogas Ilícitas; Salud Escolar.

\section{Introdução}

A adolescência é a etapa da vida compreendida entre a infância e a fase adulta, marcada por um complexo processo de crescimento e desenvolvimento biopsicossocial. É uma época de grandes transformações, de descobertas, de rupturas e de aprendizado e, por isso mesmo, uma fase que envolve riscos, medos, amadurecimento e experimentações de muitos dos comportamentos adultos.

Dentre as numerosas discussões que envolvem essa fase da vida, vem se somar o uso de drogas em sua ampla gama de possibilidades, desde as lícitas, como álcool e tabaco, até as ilícitas, como maconha, cocaína e ecstasy para recreação ou festas, entre muitas outras ${ }^{(1)}$. Sabe-se que o uso dessas substâncias é fator desencadeador de acidentes, violência, suicídios, gravidez precoce e doenças de transmissão sexual.

O Estatuto da Criança e do Adolescente considera adolescente, o individuo na faixa etária de 12 a $18 \operatorname{anos}^{(2)}$. Em 2011, havia no Brasil cerca de 78,5 milhões de crianças, adolescentes e jovens, de acordo com a Pesquisa Nacional por 
Amostra de Domicílios (PNAD), realizada pelo Instituto Brasileiro de Geografia e Estatística ${ }^{(3)}$.

Esse número corresponde a, aproximadamente, $40,2 \%$ do total da população, sendo $15 \%$ pertencentes à faixa etária de 6 a 14 anos e 5,4\% com idade de 15 a 17 anos. A grande maioria dessas crianças e adolescentes, ainda segundo o IBGE, frequenta a escola: $97,2 \%$ e $83,7 \%$, para as faixas etárias de 6 a 14 anos e de 15 a 17 anos, respectivamente ${ }^{(3)}$. Assim, é necessário considerar a escola um espaço privilegiado para implementação de políticas públicas, como as voltadas à saúde coletiva, para pessoas nessa faixa etária.

Dentro desse contexto, o Ministério da Saúde e o IBGE constituíram parceria para a realização da segunda Pesquisa Nacional de Saúde do Escolar (PeNSE) em 2012, com o intuito de descrever as prevalências dos fatores de risco e proteção de doenças crônicas não transmissíveis em adolescentes, visando orientar políticas públicas de promoção da saúde nesse grupo etário ${ }^{(4)}$.

Neste trabalho, apresentamos, interpretamos e analisamos os resultados da PeNSE, disponíveis publicamente, relacionados ao consumo de álcool e drogas ilícitas na cidade de GoiâniaGO. Essas informações poderão auxiliar na elaboração de propostas de intervenção no âmbito das Políticas Públicas de Atenção à Saúde dos adolescentes.

\section{Material e métodos}

Trata-se de um estudo transversal quantitativo realizado a partir da base de dados da Pesquisa Nacional de Saúde do Escolar, disponível para pesquisa no site do Instituto Brasileiro de Geografia e Estatística ${ }^{(5)}$.

As fontes de dados secundários de saúde, que se restringiam a estatísticas agregadas divulgadas por meio de relatórios técnicos, passaram a ser disponibilizadas sob a forma de bases eletrônicas, contendo microdados individuais, trazendo mais flexibilidade para a realização de análises descritivas e a exploração de hipóteses causais $^{(6)}$.

A PeNSE, em sua segunda edição 2012, investigou os fatores de risco e proteção à saúde dos adolescentes junto aos estudantes do 9o ano do Ensino Fundamental, ampliando sua abrangência das 26 capitais e do Distrito Federal, investigados em 2009, para uma representação do conjunto do País e Grandes Regiões, além de manter a representatividade para as capitais e $o$ $\mathrm{DF}^{(5)}$.

A pesquisa foi realizada com amostra probabilística por estratificação geográfica. O tamanho da amostra foi calculado para fornecer estimativas de proporções (ou prevalências) de algumas características de interesse, em cada um dos estratos geográficos, com um erro máximo de 0,03 em valor absoluto no nível de confiança de $95 \%{ }^{(4)}$.

O 9 $9^{\circ}$ ano do Ensino Fundamental foi escolhido por concentrar faixa etária de 13 a 15 anos, com alunos possuidores de habilidades necessárias para responder ao questionário autoaplicável e pertencentes a um grupo vulnerável, portanto, susceptíveis à exposição de diversos fatores de risco. Além do mais, a Organização Mundial da Saúde - OMS, preconiza essa faixa etária como referência para os estudos de adolescentes escolares $^{(4)}$.

Neste estudo nos limitamos à análise de alguns dados referentes ao consumo de álcool e drogas ilícitas no município de Goiânia, capital do estado de Goiás. Responderam ao questionário 3.044 escolares matriculados em uma das 77 escolas pesquisadas no município ${ }^{(4)}$.

Essas informações, por meio das quais construímos tabelas comparativas, foram obtidas no Banco de dados agregados do Sistema IBGE de Recuperação Automática - SIDRA, disponível pela internet ${ }^{(5)}$.

O objetivo dessa base de informação é armazenar tabelas com dados agregados das pesquisas que o IBGE realiza. Estes podem ser obtidos, por exemplo, pelo somatório dos valores de quesitos contidos em um questionário respondido pelos informantes da pesquisa e está associado às unidades de um nível territorial (unidade da federação, município etc.), a um período de tempo e, muitas vezes, a um conjunto de classificações que o qualificam ${ }^{(5)}$.

Na página da PeNSE 2012 no site do IBGE, na seção cigarro, álcool e outras drogas foram selecionadas: a tabela 3702 - Dados percentuais de escolares frequentando o 9o ano do Ensino Fundamental, relativos ao uso de bebida alcoólica, por sexo e dependência administrativas da escola; e a Tabela 3703 - Percentual de escolares 
frequentando o 9ọ ano do Ensino Fundamental que usaram drogas ilícitas alguma vez, por sexo e dependência administrativa da escola.

As varáveis analisadas foram: Experimentação e consumo atual de bebidas alcoólicas, episódios de embriaguez; 2) Experimentação e consumo atual de drogas ilícitas. A unidade territorial selecionada foi o município de Goiânia-GO

\section{Resultados}

Experimentação de bebida alcoólica entre os escolares

A pesquisa considerou experimentação o fato de o participante ter tomado pelo menos uma vez algum tipo de bebida alcoólica, como cerveja, chope, vinho, cachaça, pinga, vodca, vodca-ice, uísque, etc. A prevalência de escolares que responderam ter experimentado bebida alcoólica pelo menos uma vez foi de $71,9 \%$. Entre as meninas, esse percentual foi maior que entre os meninos (Tabela 1). Nas escolas privadas, o percentual de experimentação foi maior do que nas escolas públicas (Tabela 2).

Tabela 1 - Percentual de escolares frequentando o $9^{\circ}$ ano do Ensino Fundamental que experimentaram bebida alcoólica pelo menos uma vez, consumiram bebida alcoólica ao menos um dia nos últimos 30 dias e sofreram pelo menos um episódio de embriaguez, conforme o sexo. Goiânia, GO, Brasil, 2012.

\begin{tabular}{lcc}
\hline Variável & Masculino & Feminino \\
\hline Experimentação & 69,2 & 74,7 \\
Consumo atual & 26,1 & 29,5 \\
Embriaguez & 25,3 & 27,5 \\
\hline
\end{tabular}

Fonte: IBGE - Pesquisa Nacional de Saúde do Escolar.

Tabela 2 - Percentual de escolares frequentando o $9^{\circ}$ ano do Ensino Fundamental que experimentaram bebida alcoólica pelo menos uma vez, consumiram bebida alcoólica ao menos um dia nos últimos 30 dias e sofreram pelo menos um episódio de embriaguez, conforme o tipo de escola. Goiânia, GO, Brasil, 2012.

\begin{tabular}{lcc}
\hline Variável & Privada & Pública \\
\hline Experimentação & 77,2 & 69,3 \\
Consumo atual & 28,5 & 27,4 \\
Embriaguez & 23 & 28 \\
\hline
\end{tabular}

Fonte: IBGE - Pesquisa Nacional de Saúde do Escolar.
Consumo atual de bebidas alcoólicas entre os escolares

O consumo recente de bebida alcoólica entre os escolares frequentando o $9^{\circ}$ ano do Ensino Fundamental, avaliado pelo consumo feito nos últimos 30 dias de ao menos um copo ou uma dose, foi de $27,8 \%$ no geral, mas no sexo feminino tivemos uma proporção maior do que no sexo masculino (Tabela 1). Nas escolas públicas, a proporção foi ligeiramente menor do que nas escolas privadas (Tabela 2).

Episódios de embriaguez entre os escolares

Para avaliar os episódios de embriaguez, os participantes responderam à seguinte pergunta: "Na sua vida, quantas vezes você bebeu tanto que ficou realmente bêbado (a)?". No geral, $26,4 \%$ dos escolares afirmam ter sofrido pelo menos um episódio de embriaguez. No sexo feminino, a proporção foi maior do que no sexo masculino (Tabela 1). Nas escolas públicas, a proporção foi maior do que nas escolas privadas (Tabela 2).

Uso de drogas ilícitas entre os escolares

Os participantes foram questionados se haviam usado algum tipo de droga ilícita pelo menos uma vez, tal como: maconha, cocaína, crack, cola, loló, lança perfume, ecstasy. Houve uma predominância no sexo masculino em relação ao feminino (Tabela 3 ) e em escolas públicas em relação às privadas (Tabela 4).

Tabela 3 - Percentual de escolares frequentando o $9^{\circ}$ ano do Ensino Fundamental que usaram drogas ilícitas pelo menos uma vez, conforme o sexo. Goiânia, GO, Brasil, 2012.

\begin{tabular}{lcc}
\hline Variável & Masculino & Feminino \\
\hline Experimentação & 10,7 & 8,8 \\
\hline
\end{tabular}

Fonte: IBGE - Pesquisa Nacional de Saúde do Escolar.

Tabela 4 - Percentual de escolares frequentando o $9^{\circ}$ ano do Ensino Fundamental que usaram drogas ilícitas alguma vez, conforme tipo de escola. Goiânia, GO, Brasil, 2012.

\begin{tabular}{lcc}
\hline Variável & Privada & Pública \\
\hline Experimentação & 10,9 & 7,6 \\
\hline
\end{tabular}

Fonte: IBGE - Pesquisa Nacional de Saúde do Escolar. 


\section{Discussão}

Uso de bebidas alcoólicas de acordo com o sexo e tipo de escola

O uso de drogas na adolescência se dá em função de vários aspectos: a curiosidade, a falta de maturidade e de informação, o modelo parental social, os mitos e a expectativa do efeito, a pressão da indústria e da mídia, além de outros contextos ${ }^{(7)}$.

As bebidas alcoólicas são as substâncias psicotrópicas mais utilizadas por adolescentes no Brasil e no mundo. Esse consumo está inserido na nossa cultura há muitos anos, e esse comportamento conta, de fato, com ampla aceitação social, sendo valorizado até em vários aspectos socioculturais ${ }^{(8-9)}$.

Os resultados desta pesquisa apontam altas proporções de utilização precoce de álcool entre os adolescentes. Considerando que a pesquisa foi realizada com indivíduos na faixa etária dos 13 aos 15 anos e que no Brasil é proibida a venda de bebidas alcoólicas a menores de 18 anos segundo o Estatuto da Criança e do Adolescente ${ }^{(2)}$, trata-se de um uso precoce e indevido.

Entre os escolares que consumiram bebida alcoólica 30 dias antes de responder ao questionário da PeNSE, as formas mais comuns de obtê-la foi em festas, com amigos, ou comprando no mercado, loja, bar ou supermercado, respectivamente. Outros dos escolares adquiriram bebida alcoólica para o consumo, durante o período considerado, na própria casa ${ }^{(4)}$.

Um levantamento do uso de drogas entre indivíduos de primeiro e segundo graus em dez capitais brasileiras, mostrou que o início de uso de drogas pelos adolescentes se dá por volta de 12,5 e 12,8 anos para álcool e tabaco. Para outras drogas, o início de consumo é de 13,1 anos para os adolescentes e de 14,4 anos para as adolescentes. A iniciação precoce do uso dessas substâncias pode causar consequências devastadoras para a vida adulta desses adolescentes. Quanto mais precoce a experimentação, piores serão as consequências e maior o risco de desenvolvimento de abuso e de dependência de álcool( ${ }^{(7-10)}$.

O consumo precoce também foi detectado na cidade de Cajazeiras - PB, corroborando os dados levantados. Esse fato deve ser levado em conta na escolha dos momentos mais adequados ao início de abordagens preventivas ${ }^{(11)}$.
O aumento da experimentação, consumo atual e episódios de embriaguez entre as meninas parece ser resultado das mudanças de comportamento que vêm ocorrendo nas últimas décadas, quando elas passaram a ter mais liberdade para frequentar lugares e festas antes restritos aos adolescentes do sexo masculino.

Essa modificação no consumo de álcool pelo sexo feminino é considerada prejudicial devido a peculiaridades fisiológicas das mulheres. Estas apresentam níveis sérios da enzima álcool-desidrogenase mais baixos, maior proporção de gordura em relação à água corporal e variações da metabolização do álcool nas diferentes fases do ciclo menstrual. Tais características podem resultar em dependência química mesmo em menores quantidades e com maior efeito deletério ao álcool quando comparadas aos homens ${ }^{(12)}$.

A prevalência maior de experimentação e consumo atual de bebidas alcoólicas entre os alunos de escolas privadas parece estar relacionada ao maior poder aquisitivo tanto para a compra da bebida quanto para frequentar festas e baladas, embora possamos dizer que as bebidas alcoólicas estejam acessíveis a todas as classes sociais. Corroborando com esse resultado, um estudo realizado na cidade de Boa Vista, no ano de 2008, observou que os alunos de escolas privadas têm um contato mais precoce com as bebidas alcoólicas em relação aos de escola pública ${ }^{(13)}$.

Embora a experimentação e o consumo atual de bebidas alcoólicas entre os alunos de escolas particulares tenham se revelado maiores, a prevalência de episódios de embriaguez se apresenta maior entre os alunos de escolas públicas. Esse fato precisa ser elucidado por outros estudos.

Alguns estudos evidenciam que os meninos apresentam taxas de "beber pesado" maiores do que as das meninas. As pesquisas apontam que os adolescentes de ambos os sexos consomem praticamente os mesmos tipos de bebidas, com as mesmas frequências, consumindo apenas quantidades diferentes, e os meninos parecem ingerir mais que as meninas ${ }^{(7-8)}$.

Uso de drogas ilícitas

O termo drogas foi aplicado aqui considerando a mesma forma utilizada na linguagem mais comum como substâncias psicoativas ilícitas 
(maconha, cocaína, crack, heroína, LSD, ecstasy etc.), cujo uso é tido necessariamente como abusivo e que são alvo dos regimes de controle e proibição. O uso dessas substâncias também foi considerado alto entre os escolares de Goiânia, com maior prevalência de consumo entre os escolares do sexo masculino e entre os escolares das escolas públicas.

O aumento da experimentação de drogas entre jovens tem se tornado um sério problema em muitos países. A droga ilícita mais consumida na Europa e nos Estados Unidos é a maconha. O uso de Cannabis entre jovens pode ser um preditivo de desajustes psicossociais e elevada chance de dependência na vida adulta ${ }^{(4)}$.

Muitos estudos apontam outras relações entre o uso de drogas e comportamento de risco para HIV. As práticas sexuais na maioria das vezes são desprotegidas, já que a intoxicação diminui a vigilância e o julgamento, prejudicando a adoção de práticas mais seguras como o uso de camisinha $^{(1)}$.

O consumo de substâncias psicoativas constitui fenômeno relativamente frequente, sobretudo entre os jovens. Um grande número de pessoas experimenta tais substâncias. Destes, uma parcela considerável passa a fazer uso ocasional na maior parte das vezes, sem consequências danosas. Uma pequena parte desses usuários ocasionais passa para padrões de uso de risco, e alguns deles podendo se tornar dependentes ${ }^{(14)}$.

A PeNSE 2009 identificou que a experimentação de drogas ilícitas foi de 8,7\% para o conjunto dos alunos pesquisados nos Municípios das Capitais $^{(15)}$. Em 2012, a proporção para esse indicador, entre os adolescentes que frequentavam o $9^{\circ}$ ano do Ensino Fundamental em escolas dos Municípios das Capitais foi de 9,9\%, representando um ligeiro aumento em relação ao resultado observado há três anos ${ }^{(4)}$. A evidência desse aumento não pode ser negligenciada.

Os resultados da PeNSE 2012 também mostraram que, considerando exclusivamente os escolares que usaram drogas ilícitas pelo menos uma vez, 34,5\% utilizaram maconha e $6,4 \%$ consumiram crack nos 30 dias que antecederam a pesquisa. Em relação ao conjunto de escolares frequentando o $9^{\circ}$ ano do Ensino Fundamental, $0,5 \%$ relataram o uso de crack no período ${ }^{(4)}$. Considerando o quão esses dois tipos de drogas são nocivos à saúde, faz-se necessária a urgente tomada de medidas para reverter esse quadro.

\section{Conclusão}

Os resultados da pesquisa e da análise reforçam a importância que devemos dar à experimentação e consumo de álcool e outras drogas na adolescência. O aumento do consumo e a iniciação cada vez mais precoce comprovam que nossos jovens estão expostos a inúmeros fatores de risco para a saúde. Os altos percentuais aqui apresentados permitem conhecer a prevalência de alguns desses fatores nesse grupo etário, gerando evidências para se orientar a implementação de políticas públicas de promoção da saúde e prevenção do uso de substâncias psicoativas.

Há que se levar em conta também as particularidades detectadas em relação ao sexo, faixa etária e ao tipo de escola ao se pensar em ações preventivas e de promoção da saúde no âmbito escolar. Trata-se de um problema complexo que deve ser tratado de maneira complexa. Pensar um modelo único e absoluto de prevenção do uso de substâncias psicoativas entre os adolescentes é ainda um desafio diante de tantos modelos existentes.

Sabemos que os resultados efetivos de qualquer política de prevenção ao uso e abuso de substâncias psicoativas só serão observados a médio e longo prazos; portanto, as intervenções educativas precisam ser articuladas intersetorialmente de maneira continuada, respeitando o contexto e as particularidades de cada região e de cada grupo. As parcerias entre os diversos setores da sociedade, como saúde, educação, assistência social, segurança pública, esporte e lazer, entre outras, ganham especial importância no enfrentamento desse problema dentro do que preconiza a Política Nacional de Promoção da saúde.

\section{Referências}

1. Simões PM. Adolescência e Uso de drogas. In: Silveira DX, Moreira FG, organizadores. Panorama atual de drogas e dependências. São Paulo: Atheneu; 2006. p. 281-8.

2. Lei $n^{\circ} 8.069$ de 13 de julho de 1990 (BR). Estatuto da criança e do adolescente. Brasília: 
Senado Federal, Subsecretaria de Edições Técnicas; 2005.

3. Instituto Brasileiro de Geografia e Estatística. Síntese de indicadores sociais - uma análise das condições de vida da população brasileira. Rio de Janeiro; 2012.

4. Instituto Brasileiro de Geografia e Estatistica. Pesquisa Nacional de Saúde do escolar (PeNSE) 2012. Rio de Janeiro; 2013.

5. Instituto Brasileiro de Geografia e Estatística. Banco de dados agregados: sistema IBGE de recuperação automática SIDRA [Internet]. Last updated on Wednesday, November 15, 2013. [acesso 17 nov 2013]. Disponível em: http:// www.sidra.ibge.gov.br/bda/pesquisas/pense/default.asp? $\mathrm{o}=23 \& \mathrm{i}=\mathrm{P}$.

6. Coeli CM. Sistemas de Informação em Saúde e uso de dados secundários na pesquisa e avaliação em saúde. Cienc Saúde Coletiva. 2011;16,Supl 1:1211-20.

7. Marques ACPR. O adolescente usuário de drogas psicotrópicas no Brasil. In: Silva GL, organizador. Drogas: Políticas e Práticas. São Paulo: Roca; 2010. p. 27-36.

8. Pinsky I. Publicidade de bebidas alcoólicas e os jovens. São Paulo (SP): Fapesp; 2009. p.01-14.

9. Noto AR. Os índices de consumo de psicotrópicos entre adolescentes no Brasil. In: Pinsky I, Bessa MA, organizadores. Adolescência e drogas. São Paulo: Contexto; 2009. p. 45-53.

10. Machado NG, Moura ERF, Conceição MAV, Guedes TG. Uso de drogas e a saúde sexual de adolescentes. Rev Enferm UERJ. 2010;18(2):285-90.

11. Cerqueira GS, Lucenta CT, Gomes ATM, Freitas APF, Rocha NFM, Mariz SR. Consumo de álcool entre estudantes de uma escola pública da cidade de Cajazeiras, pb. SMAD, Rev. Eletrônica Saúde Mental Álcool Drog. (Ed. port.) 2011 [acesso 14 jan 2012];7(1):18-24.

12. Strauch ES, Pinheiro RT, Silva RA, Horta BL. Uso de álcool por adolescentes: estudo de base populacional. Rev Saúde Pública. [Internet]. ago 2009 [acesso 21 ago 2011]; 43:647-55.

13. Chagas LM, Vale JC, Carmo AG, Oliveira MS, Oliveira TS. O consumo de álcool entre adolescentes das escolas públicas e privadas em Boa Vista - Roraima. Norte Científico. [Internet]. dez 2008 [acesso 21 ago 2011];
3(1):89-100. Disponível em: http://www.ifrr. edu.br/SISTEMAS/revista/index.php/revista/ article/view/79.

14. Silveira DX, Moreira FG. Reflexões preliminares sobre a questão das substâncias psicoativas. In: Silveira DX, Moreira FG, organizadores. Panorama atual de drogas e dependências. São Paulo: Atheneu; 2006. p. 3-7.

15. Instituto Brasileiro de Geografia e Estatística. Pesquisa Nacional de Saúde do escolar (PeNSE) 2009. Rio de Janeiro; 2009. 